\title{
Assessing Effects of Freeze-Thaw on Biotinylated Macromolecules Using Gyrolab $^{\mathrm{TM}}$
}

Xiaodong F Liu ${ }^{1 *}$, Roni Weaver ${ }^{1}$, Charles Hottenstein ${ }^{2}$, Matthew Szapacs ${ }^{2}$, Lee Abberley ${ }^{2}$, LingSing Chen ${ }^{1}$ and Xun Wang ${ }^{1}$

${ }^{1}$ Translational Medicine Department, QPS, One Innovation Way, Suite 200, Newark, DE 19711, USA

${ }^{2}$ GlaxoSmithKline DMPK, 709 Swedeland Rd, King of Prussia, PA 19406

\begin{abstract}
Due to high affinity between biotin and avidin, biotinylation is widely used in ligand-binding-assay development for large-molecule bioanalysis. However, biotinylation adds biotin/spacer moiety onto the molecule and may affect the functional activity of the labeled molecule. The current Gyrolab ${ }^{\mathrm{TM}}$ immunoassay system requires the capture reagent to be biotinylated as the solid phase comprises a streptavidin-coated-bead column. During method development of a Gyrolab assay for quantification of otelixizumab, we discovered that the response and assay sensitivity was affected by freeze-thaw, which might be related to the type of spacers used between the biotin and the labeled antibody. The hydrophilic Polyethylene Glycol (PEG) spacer enhances water solubility but might be more liable to freeze-thaw compared to antibodies labeled with reagents having only hydrocarbon spacers. The overall response increased 10 fold after 8-hour incubation of the PEG-reagent at benchtop after thaw. The signal-to-background increased 8 fold for the same treatment. In contrast, no significant change upon freeze-thaw was observed for reagents with hydrocarbon spacers. Since Gyrolab assays do not require prolonged incubation, it provides an effective tool for assessing critical reagents in assay development and optimization, especially for evaluating time-dependent parameters for immunoassays.
\end{abstract}

Keywords: Gyrolab; Bioanalysis; Biotinylation; Linker; Spacer; Freeze-thaw; Immunoassay

Abbreviations: BSA: Bovine Serum Albumin; HABA: 4'-Hydroxyazo-Benzene-2-Carboxylic Acid; IQ: Installation Qualification; LIMS: Laboratory Information Management System; MRD: Minimum Required Dilution; OQ: Operational Qualification; PBS: Phosphate-Buffered Saline; PBST: Phosphate-Buffered Saline With 0.01\% Tween-20; PEG: Polyethylene Glycol; PK: Pharmacokinetics; PMT: Photomultiplier-Tube; PQ: Performance Qualification; TK: Toxicokinetics

\section{Introduction}

Recent advances in medicine reveal that the immune system plays a role in reduction of insulin producing cells and that Type 1 diabetes is an autoimmune disease. Thus, immune-suppression may be a promising disease-modifying approach to correct insufficient production of insulin [1-3]. Otelixizumab (GSK2136525, TRX4, ChAglyCD3) is a chimeric humanized monoclonal antibody which is an investigational immune-modulatory drug targeting CD3 and reducing $\mathrm{T}$ cell activation and cytokine release and is being developed for the treatment of Type 1 diabetes and other autoimmune diseases $[4,5]$. The technology used in this study, Gyrolab ${ }^{\mathrm{TM}}$, represents a recent breakthrough for large molecule bioanalysis to support biologic drug development. The advantages of this innovative platform include fully automated nanoscale immunoassay capability, better assay reproducibility and data quality, small reagent and sample volumes, no cross-talk and hook effect, and rapid assay development and validation as a result of reduced run time. Gyrolab has been increasingly used in pharmaceutical industry for immuno-bioanalysis. A fully validated Gyrolab assay for large molecule Pharmacokinetic (PK) or Toxicokinetic (TK) bioanalysis has been reported recently [6].

At present, the Gyrolab immunoassay system requires the capture reagent to be biotinylated as the solid phase comprises a streptavidincoated-bead column. The performance of a Gyrolab assay relies on properties of the biotinylated molecule. Biotinylation adds biotin/ spacer moiety onto the molecule and may affect the functional activity of the labeled molecule. In this study, we report that freeze-thaw of a biotinylated reagent and probably the type of biotin spacers affected the overall response and assay sensitivity. With proper treatment and careful selection of biotinylated reagents, variability in assay performance could be reduced. Furthermore, Gyrolab was shown in this study to be a useful tool in evaluating properties of biotinylated molecules.

\section{Materials and Methods}

The Gyrolab workstation used in this study, including the interface with Watson laboratory information management system (LIMS), was fully validated through the process of Installation Qualification (IQ), Operational Qualification (OQ), and Performance Qualification (PQ) to meet the requirement for compliance with 21 CFR Part 11. Gyrolab Bioaffy $^{\mathrm{TM}}$ CD contains streptavidin-bead packed microstructures. Reagents and samples were delivered separately to the microstructures through a volume-defined nanofluidic system. Otelixizumab was captured on the microstructure by a biotinylated monoclonal antibody and then detected by an Alexa-labeled monoclonal antibody.

The capture antibody $(\mathrm{AbT})(\mathrm{GSK})$ was biotinylated using EZLink $^{\circledR}$ NHS-PEO Solid Phase Biotinylation kit (Thermo Scientific Cat\# 21450) following manufacturer's instructions. The antibody

*Corresponding author: Xiaodong F Liu, QPS, One Innovation Way, Suite 200, Newark, DE 19711, USA, Tel: + 1302453 5946; Fax: + 1302737 3759, E-mail: frankliu8@yahoo.com

Received October 25, 2014; Accepted November 09, 2014; Published November 13,2014

Citation: Liu XF, Weaver R, Hottenstein C, Szapacs M, Abberley L, et al. (2014) Assessing Effects of Freeze-Thaw on Biotinylated Macromolecules Using Gyrolab $^{\text {TM }}$. J Bioanal Biomed 6: 049-051. doi:10.4172/1948-593X.1000110

Copyright: @ 2014 Liu XF, et al. This is an open-access article distributed under the terms of the Creative Commons Attribution License, which permits unrestricted use, distribution, and reproduction in any medium, provided the original author and source are credited. 
was first buffer-exchanged with phosphate-buffered saline (PBS) on a Nanosep $^{\circledR}$ 30K OMEGA ${ }^{\mathrm{TM}}$ Centrifugal Device (Pall Life Sciences Cat\# OD030C33) before biotinylation. For the biotin spacer comparison (Figure 1), the AbT was also biotinylated using EZ-Link Sulfo-NHSLC-Biotin (Thermo Scientific Cat\# 21327) following manufacturer's instructions, both at a challenge molar ratio of 12:1 (biotin: protein). The biotin/protein ratio of the produced conjugate was determined by the 4'-hydroxyazo-benzene-2-carboxylic acid (HABA) assay using EZ ${ }^{\mathrm{TM}}$ Biotin Quantitation Kit (Thermo Scientific Cat\# 28005) following manufacturer's instructions. The biotin/protein ratios of BiotinPEG4-AbT and Biotin-LC-AbT were 4.4 and 5.4, respectively. Protein concentration was determined by absorbance at $280 \mathrm{~nm}$ using 1.35 as the molar extinction coefficient $(\varepsilon)$ in $\mathrm{cm}^{-1}(\mathrm{mg} / \mathrm{mL})^{-1}$ of a typical IgG [7]. The detection antibody $(\mathrm{AbH})(\mathrm{GSK})$ was labeled with Alexa Fluor $^{\mathbb{B}}, 100 \mu \mathrm{g}$ antibody per batch, using Alexa Fluor 647 Labeling Kit (Life Tech Cat\# A-20186) following manufacturer's instructions. The reagent was buffer-exchanged with $0.1 \mathrm{M}$ bicarbonate solution prior to labeling. The Alexa-labeled $\mathrm{AbH}$ with an Alexa/protein ratio of 7.7 was used for the study. The biotin and Alexa labeled proteins were stored in the presence of $0.1 \%$ Bovine Serum Albumin (BSA) and 0.01\% sodium azide at either $4^{\circ} \mathrm{C}$ for up to one month or stored at $-20^{\circ} \mathrm{C}$ in aliquots for up to one year.

The assay conditions were evaluated and optimized on a Bioaffy $1000 \mathrm{CD}$, which provided 2-4 fold more sensitivity than a Bioaffy $200 \mathrm{CD}$ for the assay. The combination of $150 \mu \mathrm{g} / \mathrm{mL}$ of the capture and $25 \mathrm{nM}$ of the detection reagents provided the highest signalto-background ratio when the Biotin-PEG4-AbT was used as the capture reagent. This condition was also used for the two capture reagent comparison. The capture reagents were diluted in PhosphateBuffered Saline with $0.01 \%$ Tween-20 (PBST). The detection reagent was centrifuged at $16000 \mathrm{xg}$ for $2 \mathrm{~min}$ and diluted in Rexxip $\mathrm{F}^{\mathrm{TM}}$ buffer (Gyros). Otelixizumab (12 mg/mL, GSK) was aliquoted and stored at $-70^{\circ} \mathrm{C}$. Calibration standards were prepared at the concentrations of $2.5,10,35,125,500,2000,7500 \mathrm{ng} / \mathrm{mL}$ by spiking otelixizumab in pooled human serum (Bioreclamation) with at least $95 \%$ matrix in the final volume and were stored at $-70^{\circ} \mathrm{C}$. All samples in neat human sera were diluted 5 fold in Rexxip $\mathrm{H}^{\mathrm{TM}}$ buffer (Gyros) before loaded on $\mathrm{CD}$. Thus, the on-CD concentrations for the standards were $0.5,2,7$, $25,100,400,1500 \mathrm{ng} / \mathrm{mL}$, respectively. All frozen standards (stored at $-70^{\circ} \mathrm{C}$ ) and reagents (stored at $-20^{\circ} \mathrm{C}$ ) were thawed unassisted at room temperature. Data acquisition at $1 \%$ Photomultiplier-Tube (PMT) level was found appropriate for the assay conditions described above. Regression was performed by Gyrolab Evaluator (v 3.1.5.137, Gyros $\mathrm{AB}$, Sweden) with 5-parameter logistic fit without including the blank. Weighting was applied for response $(1 / \mathrm{Y})$.

\section{Results and Discussion}

A Gyrolab assay for otelixizumab in human serum was developed
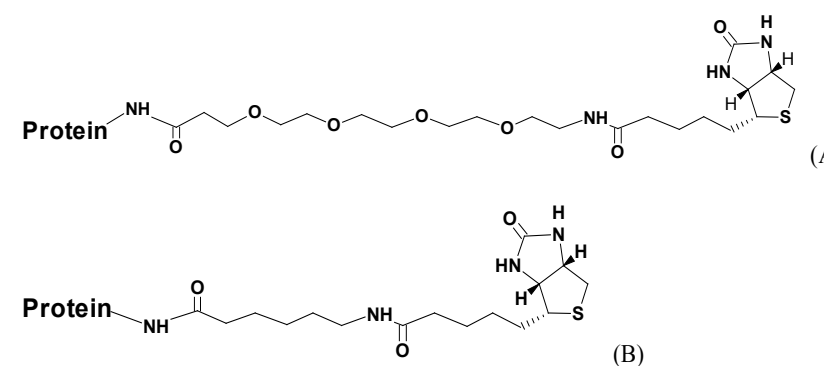

Figure 1: Structures of Biotin-PEG4-AbT (A) and Biotin-LC-AbT (B).

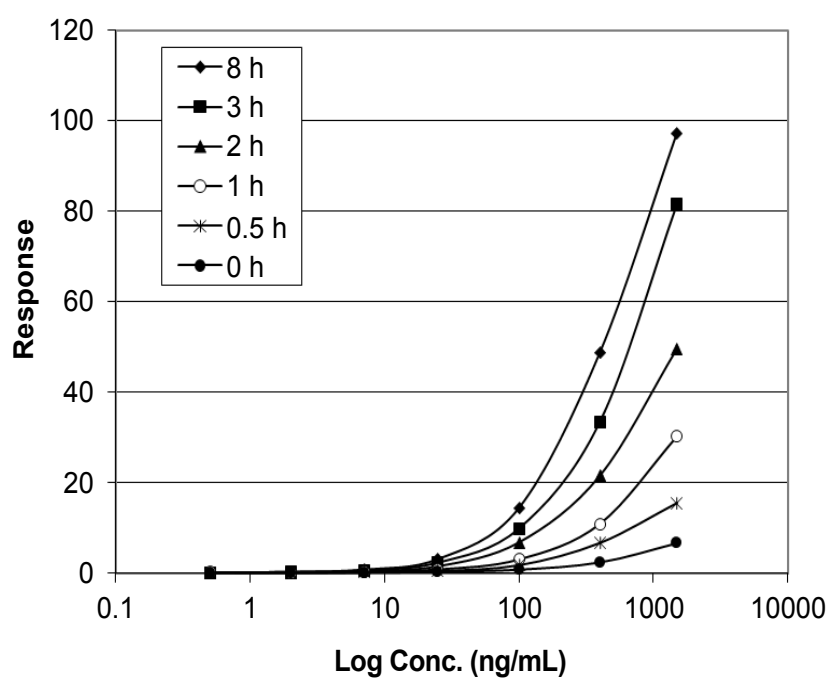

Figure 2: Increasing activity of Biotin-PEG4-AbT after freeze-thaw. BiotinPEG4-AbT was stored at $-20^{\circ} \mathrm{C}$ for at least 24 hours and then incubated at room temperature for $0.5,1,2,3$, and 8 hours before use or used immediately after thaw $(0 \mathrm{~h})$

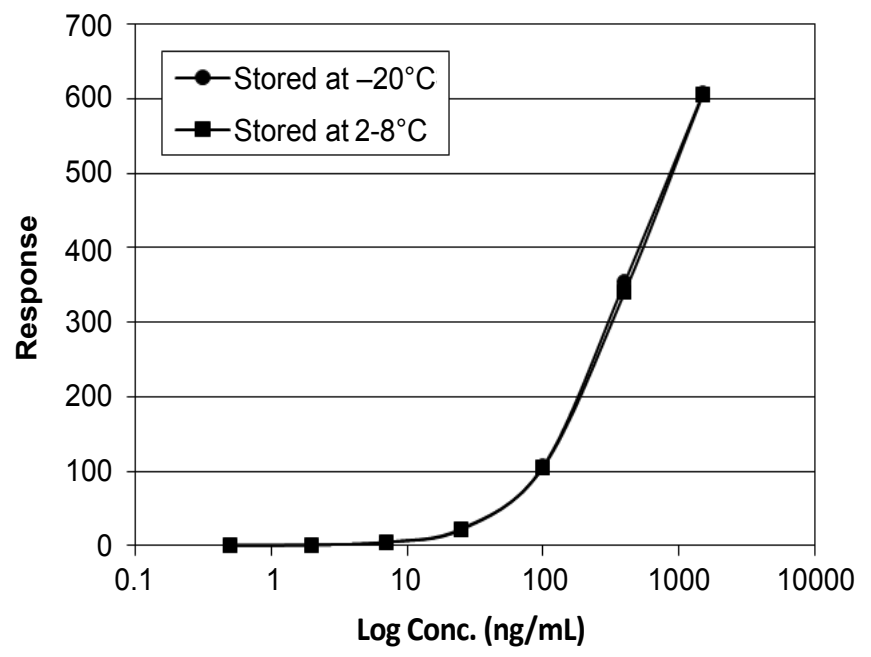

Figure 3: Compare Biotin-LC-AbT stocks with or without freeze-thaw. Biotin-LCAbT was stored at $2-8^{\circ} \mathrm{C}$ or stored at $-20^{\circ} \mathrm{C}$ for at least 24 hours and then used immediately after thaw.

with a quantification range of 2.5-2000 $\mathrm{ng} / \mathrm{mL}$ with Minimum Required Dilution (MRD) of 5. During the method development, significant variability in total response and sensitivity was observed. We noticed that the variability was related to the reagents' time at room temperature or reagents' time under light. Frozen standards, capture and detection reagents were all tested for room temperature stability. The detection reagent was tested for light sensitivity as well. The results indicate that standards and the detection reagent were all stable at room temperature under light but the activity of the BiotinPEG4-AbT capture reagent increased significantly in a time-dependent manner (Figure 2). The overall response increased 10 fold after 8-hour incubation and the signal-to-background increased 8 fold. Although the activity of the freeze-thawed reagent increased greatly at room temperature, it was still lower than the activity (a response of $\sim 270$ at 
$1500 \mathrm{ng} / \mathrm{mL}$ ) of the reagent stored at $2-8^{\circ} \mathrm{C}$ suggesting that the BiotinPEG4-AbT reagent lost activity upon freeze-thaw rather than gained additional activity.

When the same antibody was biotinylated with hydrocarbon spacers, the conjugate Biotin-LC-AbT was not affected by freezethaw (Figure 3). One explanation could be that although hydrophilic Polyethylene Glycol (PEG) spacer enhances water solubility of the biotinylated reagent its activity was more likely to be affected by freeze-thaw compared to antibodies labeled with reagents having only hydrocarbon spacers (Figure 1). Since the two capture reagents were prepared using different types of biotinylation kits, alternative labeling related interpretations for this observation cannot be ruled out.

Under the current experimental conditions, higher signals were observed for the Biotin-LC-AbT compared to the Biotin-PEG4-AbT. This could be due to different labeling methods or difference in their resulted biotin/protein ratio, where Biotin-LC-AbT had a ratio of 5:4, higher than that of Biotin-PEG4-AbT (4.4).

Since plate-based assays require prolonged and stepwise incubations before the endpoint can be reached, time-critical assay parameters cannot be easily evaluated in these assays. In contrast, Gyrolab assays can be particularly useful for assessing the time dependent properties of biotinylated reagents because data acquisition can occur minutes after reagents and samples are loaded onto the machine.

In summary, freeze-thaw of a biotinylated assay reagent could affect performance of an immunoassay. A biotinylated antibody with a PEG4 spacer might be more susceptible to freeze-thaw than a biotinylated antibody with a hydrocarbon spacer. Extended incubation periods might be necessary after thaw for a biotinylated reagent to avoid potential variability in the assay. For short-term storage of biotinylated reagents, $2-8^{\circ} \mathrm{C}$ is preferable. Since the Gyrolab assay does not require incubation, it provides an effective tool for assessing critical reagents in assay development and optimization, especially for evaluating timedependent parameters.

\section{References}

1. Herold KC, Hagopian W, Auger JA, Poumian-Ruiz E, Taylor L, et al. (2002) Anti-CD3 monoclonal antibody in new-onset type 1 diabetes mellitus. $\mathrm{N}$ Engl $\mathrm{J}$ Med 346: 1692-1698.

2. Herold KC, Taylor L (2003) Treatment of Type 1 diabetes with anti-CD3 monoclonal antibody: induction of immune regulation. Immunol Res 28: 141150.

3. Bisikirska BC, Herold KC (2004) Use of anti-CD3 monoclonal antibody to induce immune regulation in type 1 diabetes. Ann N Y Acad Sci 1037: 1-9.

4. Chatenoud L, Vandemeulebroucke E, Ziegler AG, Mathieu C, Kaufman L, et al (2005) Insulin needs after CD3-antibody therapy in new-onset type 1 diabetes. N Engl J Med 352: 2598-2608.

5. Kaufman A, Herold KC (2009) Anti-CD3 mAbs for treatment of type 1 diabetes Diabetes Metab Res Rev 25: 302-306.

6. Liu XF, Wang X, Weaver RJ, Calliste L, Xia C, et al. (2012) Validation of a gyrolab ${ }^{\mathrm{TM}}$ assay for quantification of rituximab in human serum. J Pharmacol Toxicol 65: 107-114.

7. Harlow E, Lane D (1988) Antibodies: a Laboratory Manual. ( $1^{\text {st }}$ Edition) Cold Spring Harbor Laboratory Publications, New York. 